\title{
Las fronteras de la pandemia: lecciones para la gobernanza y la cooperación en las ciudades de la frontera México-Estados Unidos
}

\section{The borders of the pandemic: lessons on governance and cooperation in United States-Mexico border cities}

Recibido el 13 de octubre de 2020

Aceptado el 16 de marzo de 2021.

Publicado el 26 de marzo de 2021.

*Autor para correspondencia: Francisco Lara-Valencia. Correo electrónico: francisco.lara@asu.edu Licencia Creative Commons Atribución-NoComercial 4.0 Internacional.
Francisco Lara-Valencia ${ }^{\text {* }}$ (1D) https://orcid.org/0000-0002-7686-5815
Hilda García-Pérez ${ }^{\mathrm{b}}$ (1) https://orcid.org/0000-0002-6357-4525

a Arizona State University, School of Transborder Studies, Tempe, Arizona, Estados Unidos, correo electrónico: francisco.lara@asu.edu

${ }^{\mathrm{b}}$ El Colegio de la Frontera Norte, Departamento de Estudios de Población, Nogales, Sonora, México, correo electrónico: mhgarciaperez@colef.mx

\section{Resumen}

Este artículo documenta y analiza la respuesta de las ciudades de la región fronteriza México-Estados Unidos a la emergencia sanitaria y a un proceso sustancial de refronterización desencadenado por el COVID-19. Como muchos otros países, la estrategia principal de Estados Unidos y México para contener la propagación del coronavirus ha sido la adopción de medidas de distanciamiento social y restricciones a la movilidad, incluyendo el cierre de la frontera común. Una implicación inmediata de este proceso es el reforzamiento de la presencia de los gobiernos nacionales en la gestión de la salud fronteriza y, potencialmente, la contracción de los espacios de gobernanza en los que participan tradicionalmente actores subnacionales. Mediante el análisis de datos secundarios de ocho pares de ciudades fronterizas contiguas, se examinan las respuestas de actores locales a la pandemia en el marco de un proceso acelerado de refronterización y se perfilan escenarios de gobernanza y cooperación trasfronteriza pospandemia.

Palabras clave: COVID-19, gobernanza, cooperación transfronteriza, actores locales, refronterización, frontera.

\section{Abstract}

This article documents and analyzes the response of cities in the U.s.-Mexico border region to the health emergency and a significant process of rebordering triggered by COVID-19. Like many other countries, the United States and Mexico's primary strategy to contain the spread of coronavirus has been the adoption of social distancing measures and restrictions on mobility, including the closure

CÓMO CITAR: Lara-Valencia, F. \& García-Pérez, H. (2021). Las fronteras de la pandemia: lecciones para la gobernanza y la cooperación en las ciudades de la frontera México-Estados Unidos [The borders of the pandemic: lessons on governance and cooperation in United States-Mexico border cities]. Estudios Fronterizos, 22, e067. https://doi.org/10.21670/ref.2104067 
of the common border. An immediate implication of this process is the strengthening of national governments' presence in border health management and, potentially, the contraction of governance spaces in which subnational actors traditionally participate. By analyzing secondary data for eight pairs of co-adjacent border cities, local actors' responses to the pandemic are examined in the context of a rapid rebordering process and postpandemic governance, and cross-border cooperation scenarios are explored.

Keywords: COVID-19, governance, cross-border cooperation, local actors, rebordering, border.

\section{Introducción}

La celeridad de la propagación del COVID-19 y la gravedad de la epidemia causada por el virus, ha generado una situación de crisis de escala global con afectaciones sobre todos los sistemas sociales, no solo al de la salud pública. Desde la declaratoria de emergencia internacional emitida por la Organización Mundial de la Salud (oms) el 11 de marzo de 2020, los gobiernos nacionales y subnacionales en casi todos los países del mundo han respondido a la pandemia con la implementación de una variedad de medidas para detener la propagación del virus (World Health Organization [wHo], 2020). Ante la falta de una vacuna para prevenir la propagación de esta enfermedad infecciosa, el denominador común de las medidas tomadas fue la adopción de acciones para restringir la movilidad y la interacción social. A escala internacional, los gobiernos nacionales han utilizado el cierre de fronteras como el instrumento principal para mitigar la exposición de su población y territorio a un agente infeccioso de naturaleza externa (Lara-Valencia et al., 2020). Como resultado, las fronteras nacionales han resurgido en regiones donde habían dejado de ser utilizadas como instrumentos de control territorial, o se han reforzado en aquellas donde esta función continúa siendo relevante (LaraValencia et al., 2020). En general, la pandemia del COVID-19 ha inducido un proceso de refronterización a nivel global con implicaciones multiescalares impredecibles, y sobre las cuales solo podemos especular en este momento. Para comprender mejor su significado actual y sus repercusiones en el largo plazo es necesario desempacar el proceso de refronterización identificando cada uno de sus componentes. En forma específica, este proceso involucra tres subprocesos interrelacionados.

Primeramente, la pandemia podría estar dando pie a una regresión hacia el centro en la toma de decisiones respecto a la gestión de las fronteras y su hinterland. Este giro es más evidente en regiones donde las fronteras fueron desactivadas décadas atrás como instrumentos de control territorial y se establecieron políticas para facilitar y promover la integración transfronteriza. Su efecto más inmediato podría ser la redefinición de los roles de actores locales y nacionales en la producción de las agendas fronterizas y sus prioridades, un aspecto que preocupa a proponentes de una mayor participación local en la gestión de asuntos fronterizos. En el caso de la Unión Europea, por ejemplo, la erección de fronteras internas a la movilidad en el marco de la pandemia ha generado algunas tensiones y protestas por la relegación de las autoridades locales del manejo del espacio transfronterizo y adopción de medidas unilaterales que imponen restricciones a la movilidad (Klatt, 2020).

En segundo lugar, el giro hacia el centro coincide con discursos soberanistas y nacionalistas en diferentes partes del mundo, y con una fuerte crítica a la globalización 
en años recientes. Una característica común de estas tendencias es la representación de la frontera como un escudo protector frente a amenazas externas y su necesario reforzamiento como una condición para la preservación de la integridad nacional. Este proceso tiende a transformar el lindero internacional en perímetro de seguridad y a la zona adyacente en un espacio de riesgo, lo que en la práctica refuerza la frontera geopolítica con un proceso de refronterización de tipo socio-cultural (Wille, 2020).

En tercer lugar, el resurgimiento de las fronteras nacionales como herramientas de control de una amenaza externa real o imaginaria puede contribuir a socavar las narrativas que representan a la frontera como un recurso, y que han sido fundamento de acciones de cooperación transfronteriza no solo en materia económica, sino también en términos políticos, culturales, ambientales y de salud pública. Tanto en Europa como en América del Norte, varias ciudades fronterizas han sido capaces de proyectarse como espacios de innovación y competitividad a nivel global debido a su capacidad para aprovechar diferenciales y complementariedades económicas, institucionales y culturales como resultado de su ubicación limítrofe (Sohn, 2014).

Con este panorama de fondo, este artículo explora la respuesta de las ciudades de la frontera México-Estados Unidos a las acciones de los gobiernos federales para contrarrestar la pandemia y sus efectos sobre la interacción transfronteriza. El análisis está anclado en la premisa de que durante décadas las ciudades de la frontera han establecido y consolidado formas descentralizadas para la gestión de problemas locales, especialmente en áreas relacionadas con la salud pública. Al considerar esta capacidad, podría argumentarse que la acción descentralizada debería ser la respuesta de primera instancia de las ciudades de la frontera frente a la pandemia, especialmente por el impacto de acciones centralizadas sobre la movilidad y sobre las altamente interdependientes sociedades fronterizas. El grado y el modo con el que estas expectativas se cumplen son examinadas mediante el análisis y evaluación de las acciones de actores locales - gubernamentales y no gubernamentales- en ocho pares de ciudades fronterizas contiguas. El artículo comienza con una revisión de las formas de cooperación y gestión de la salud pública observadas en la región. Posteriormente, se hace una descripción de la metodología de investigación, seguida de la presentación y discusión de los resultados. A manera de conclusión se delinean escenarios de gobernanza y cooperación a partir de la evidencia recogida por este estudio.

\section{Gobernanza de la salud en la frontera México-Estados Unidos}

Cerca de 16000000 de personas residen en los 24 condados estadounidenses y 40 municipios mexicanos adyacentes a la línea divisoria internacional compartida por ambos países (Instituto Nacional de Estadística y Geografía [Inegi], 2020; United States Census Bureau, 2020). La mayoría de esta población (85\%) vive en 15 conurbaciones binacionales, que contrastan entre sí y ocupan una posición singular en la jerarquía urbana de sus respectivos países. Las ciudades fronterizas estadounidenses son generalmente más pequeñas y presentan niveles de desarrollo urbano más altos que sus contrapartes mexicanas. Comparadas con otras ciudades en su propio país, la mayoría de las ciudades fronterizas estadounidenses se caracterizan por sus altos niveles de desempleo y pobreza, y por una población predominantemente mexicana en su origen. En contraste, las ciudades fronterizas mexicanas son más afluentes que 
el promedio nacional y experimentan niveles de empleo también mayores, pero se distinguen por sus carencias urbanas y falta de planeación. Un aspecto compartido por las ciudades de la frontera es su caracterización recurrente en los medios de comunicación de cada país como territorios fértiles para la ilegalidad, la violencia, la degradación ambiental y la transmisión de enfermedades infecciosas (Arreola, 2010; Johnson \& Niemeyer, 2008).

En virtud de las profundas diferencias económicas y sociales que existen entre México y Estados Unidos, la región fronteriza ha sido descrita como la región limítrofe con las desigualdades estructurales más profundas del mundo contemporáneo (Grimson, 2006; Varady \& Mack, 1995). Aunque históricamente la principal función de la frontera entre los dos países ha sido la separación y la distinción, la frontera también es un espacio de movilidad e interacción social intensas (Álvarez, 2012). En consecuencia, la región fronteriza es asimismo un espacio donde el gobierno federal tiene una presencia constante por su participación en el diálogo y negociación bilateral, y formulación e implementación de políticas que intentan dar orden a la intensa relación entre los dos países en materia económica, migratoria, ambiental y de seguridad nacional (Ganster \& Collins, 2017). La región también se significa por la existencia de un tejido social transfronterizo crecientemente denso, que resulta de una población transnacional que vive, trabaja y se recrea en ambos lados de la frontera. Aunque la interacción y movilidad transfronterizas son sensibles a los ciclos económicos y a los cambios en las prioridades nacionales respecto a la región, las tendencias demográficas de largo plazo, los vínculos sociales históricos, la integración de los mercados laborales y la expansión del comercio global, alimentan un tejido transfronterizo resiliente y cada vez más diverso (Gerber et al., 2010). De hecho, durante las últimas tres décadas, la región fronteriza observó una acentuación de la trayectoria hacia la integración transfronteriza, como resultado del crecimiento de los flujos de comercio y la interacción social (Anderson et al., 2008).

\section{Cooperación transfronteriza y salud}

Aunque la frontera México-Estados Unidos es un espacio de contrastes donde las diferencias económicas, culturales e institucionales entre los dos países pueden ser fuente de tensión y conflicto, también es cierto que estas diferencias inducen el desarrollo de complementariedades e interdependencias que estimulan formas diversas de cooperación (Homedes \& Ugalde, 2003). Esto es particularmente cierto en el campo de la salud, donde existe una larga historia de intercambio y colaboración que ha resultado en expresiones de gobernanza transfronteriza con diversos grados de sofisticación y formalidad. Los hitos más significativos de esta historia son los siguientes.

Durante la primera mitad del siglo xx, el establecimiento de bases militares estadounidenses cerca de la frontera y el crecimiento de la migración internacional en el marco del Programa de Braceros (1942-1964) crearon nuevos patrones de interacción y movilidad en la frontera que elevaron la incidencia de enfermedades de transmisión sexual y otras enfermedades contagiosas como la tuberculosis (Arreola \& Curtis, 1993; Garza-Almanza, 2018). Como resultado, y a iniciativa de los Estados Unidos, la Organización Panamericana de la Salud (ops) estableció en 1942 una oficina de campo en El Paso, Texas, que operó programas de prevención contra la 
sífilis, tuberculosis y otras enfermedades contagiosas en ambos lados de la frontera (Garza-Almanza, 2018). Como observa Collins-Dogrul (2006), con la colaboración de autoridades mexicanas, la ops creó laboratorios en Ciudad Juárez, Nuevo Laredo y Mexicali y capacitó a profesionales de la salud en estas ciudades en la promoción de prácticas sanitarias a nivel comunitario y en la detección y tratamiento de estas enfermedades. En 1943 también se constituyó la Asociación Fronteriza México-Estados Unidos para la Salud (AFMEs), un organismo binacional que contaba con el apoyo de la ops, y que a su vez estimuló numerosas iniciativas de cooperación transfronteriza para el control de enfermedades transmisibles, contribuyó al desarrollo de capacidades y dio impulso a una agenda transfronteriza de salud (García-Pérez, 2007). Aunque las oficinas de campo de la ops y la AFMEs dejaron de operar en 2014, su actividad dio como resultado la creación de condiciones que han sido identificadas en los estudios sobre cooperación transfronteriza como fundamentales para el desarrollo de la cooperación y gobernanza transfronteriza. Estas condiciones incluyen arreglos formales o informales entre organizaciones y grupos cívicos locales, instituciones que facilitan el intercambio sostenido de información y de recursos necesarios para la cooperación, y gestación de una visión compartida resultante de dinámicas espaciales que refuerzan la cercanía y no la separación (Collins-Dogrul, 2006; Hataley \& Leuprecht, 2018).

Un legado de la actividad de la ops y la AFMes son los Consejos Binacionales de Salud (Cobinas). Los Cobinas fueron establecidos en 1963 durante la reunión anual de la AFMEs en Nogales, Arizona, como un mecanismo para promover la colaboración entre ciudades fronterizas y han sido descritos como "una matriz binacional de salud pública que colabora para mejorar las condiciones [de salud] en la frontera" (azdhsadmin, 2011). En la actualidad operan cerca de 16 Cobinas en los que participan autoridades locales de salud, organizaciones no gubernamentales, grupos académicos y asociaciones profesionales que se reúnen periódicamente para intercambiar información y coordinar actividades de prevención y vigilancia epidemiológica local en ambos lados de la frontera (García-Pérez, 2007, 2010).

En julio de 2000 iniciaron oficialmente las actividades de la Comisión de Salud Fronteriza México-Estados Unidos (CSFMEU). La comisión tiene como mandato mejorar los indicadores de salud de la región fronteriza, que incluye todos los municipios comprendidos dentro de una franja de 100 kilómetros que se extiende en ambos lados de la frontera (Panamerican Health Organization [Paho], 2012). Aunque criticada por las dificultades que ha tenido para estructurar una agenda de salud regional y la falta de actividades programáticas continuas, la comisión es el mecanismo oficial responsable de canalizar la cooperación binacional relativa a la frontera (CollinsDogrul, 2006; Homedes \& Ugalde, 2003).

En suma, el aparato institucional creado por México y Estados Unidos para atender los problemas de salud de la región fronteriza compartida no tiene paralelo en ningún otro sector de la relación bilateral. El nivel de institucionalización logrado ha permitido la continuidad de la colaboración en ciertas áreas y regiones, y ha derivado en logros importantes en materia de capacitación, intercambio de información, investigación epidemiológica y vigilancia de enfermedades infecciosas como la tuberculosis y vectoriales como el dengue. Un aspecto significativo de esta institucionalización es el nivel de involucramiento de actores locales, incluidos los departamentos municipales de salud, organizaciones comunitarias, agrupaciones profesionales y universidades regionales de ambos lados de la frontera. Como se indicó, el resultado ha sido la formación de una matriz de colaboración que incluye mecanismos formales e informales 
de colaboración, que muchas veces son indistinguibles por su grado de imbricación (García-Pérez, 2007).

Aunque el grado de institucionalización cristalizado en esta estructura es insuficiente para el manejo integrado de los problemas y prioridades de salud fronterizos, su importancia no puede ser minimizada en el contexto de la actual emergencia de salud pública creada por la propagación del COVID-19 en la región.

La Tabla 1 presenta el número de casos confirmados de COVID-19 y defunciones en municipios y condados fronterizos en agosto de 2020. Al comparar dichas tasas con los datos a nivel nacional, la información muestra que los municipios y condados fronterizos han sido afectados de manera desproporcionada por la pandemia. La mayoría de los municipios seleccionados muestran una incidencia de COVID-19 mayor que el nivel nacional, tanto en el número de casos confirmados como en el número de defunciones. El mismo patrón se observa entre los condados fronterizos adyacentes, sin diferencias importantes en términos de su tamaño y ubicación en la geografía de la frontera.

Tabla 1. Casos confirmados de COVID-19 y defunciones en municipios y condados fronterizos seleccionados (9 de agosto de 2020)

\begin{tabular}{|c|c|c|c|c|c|c|c|c|c|}
\hline \multirow{2}{*}{ Municipios } & \multicolumn{2}{|c|}{ Casos } & \multicolumn{2}{|c|}{ Defunciones } & \multirow{2}{*}{ Condados } & \multicolumn{2}{|c|}{ Casos } & \multicolumn{2}{|c|}{ Defunciones } \\
\hline & Número & Tasa & Número & Tasa & & Número & Tasa & Número & Tasa \\
\hline Mexicali, B. C. & 7668 & 775.8 & 1339 & 135.5 & Imperial, CA & 9693 & 5348.9 & 244 & 134.6 \\
\hline $\begin{array}{l}\text { Ciudad Acuña, } \\
\text { Coah. }\end{array}$ & 1391 & 941.1 & 129 & 87.3 & Del Rio, TX & 1332 & 2717.0 & 32 & 65.3 \\
\hline $\begin{array}{l}\text { Ciudad Juárez, } \\
\text { Chih. }\end{array}$ & 3644 & 261.9 & 692 & 49.7 & El Paso, TX & 16308 & 1943.2 & 282 & 33.6 \\
\hline Reynosa, Tam. & 4293 & 664.3 & 415 & 64.2 & Hidalgo, TX & 19534 & 2248.6 & 638 & 73.4 \\
\hline $\begin{array}{l}\text { Nuevo. Lare- } \\
\text { do, Tam. }\end{array}$ & 1663 & 416.3 & 201 & 50.3 & Webb, TX & 7825 & 2828.5 & 96 & 34.7 \\
\hline Nogales, Son. & 1957 & 836.5 & 235 & 100.4 & Santa Cruz, AZ & 2662 & 5725.0 & 53 & 114.0 \\
\hline Ojinaga, Chih. & 98 & 349.5 & 1 & 3.6 & Presidio, TX & 46 & 686.2 & 2 & 29.8 \\
\hline $\begin{array}{l}\text { San Luis Río } \\
\text { Colorado, Son. }\end{array}$ & 1362 & 706.7 & 181 & 93.9 & Yuma, AZ & 11510 & 5383.9 & 288 & 134.7 \\
\hline México & 485831 & 406.4 & 53001 & 44.3 & $\begin{array}{l}\text { Estados } \\
\text { Unidos }\end{array}$ & 5048035 & 1537.9 & 161491 & 49.2 \\
\hline
\end{tabular}

*Tasas por 100000 residentes

Fuente: Gobierno de México (https://coronavirus.gob.mx/datos/\#COMNac) y Center for Disease Control and Prevention (CDC), Coronavirus COVID-19 (https://www.cdc.gov/coronavirus/2019-ncov/casesupdates/county-map.html)

$\mathrm{Al}$ tener como telón de fondo el nivel de institucionalización logrado por la frontera en el campo de la salud y el impacto del COVID-19 sobre la región, las siguientes secciones analizan y discuten las respuestas de algunas ciudades de la frontera a la emergencia sanitaria. 


\section{Metodología}

Los datos utilizados en este análisis son el resultado de una revisión sistemática de fuentes de información secundaria relativa a ocho pares de ciudades contiguas en la frontera entre México y Estados Unidos. Mientras en la parte occidental de la frontera se analizó información de Mexicali/Calexico, San Luis Río Colorado/Yuma y Nogales/Nogales, en el área del río Bravo se analizaron datos de Ciudad Juárez/El Paso, Ojinaga/Presidio, Ciudad Acuña/Del Río, Nuevo Laredo/Laredo y Reynosa/McAllen. El propósito de la revisión fue identificar y documentar el espectro de acciones concretas y conmensurables adoptadas por actores locales para contener la propagación del coronavirus (v. gr. cierre de negocios), o para mitigar los efectos de las medidas tomadas para controlar la incidencia del COVID-19 (v. gr. asistencia a grupos vulnerables). Los actores considerados fueron tanto entidades gubernamentales como no gubernamentales cuyo espacio de actuación es primordialmente alguna de las ciudades.

El primer paso en la revisión fue la auscultación de los sitios web oficiales de las ciudades seleccionadas buscando comunicados de prensa, anuncios públicos y documentos que dieran cuenta de decisiones tomadas por autoridades municipales en relación con la pandemia. El segundo paso fue la revisión de las páginas web de periódicos y noticieros locales, tanto para documentar las acciones oficiales como para ampliar el espectro de acciones con la incorporación de la actividad de actores no gubernamentales. Finalmente, se realizó una búsqueda abierta en internet usando los términos "COVID-19", "coronavirus" o "pandemia”, en combinación con el nombre de la ciudad seleccionada, lo que permitió ampliar aún más la cobertura de la revisión. Las acciones identificadas a través de este procedimiento fueron compiladas con la ayuda de una cédula estandarizada creada para registrar en forma sistemática el tipo de acciones, los actores implicados, el campo de actividad, así como el nivel de cooperación transfronteriza involucrado. La revisión cubrió el periodo que va del 11 de marzo al 31 de julio de 2020 y fue efectuada por estudiantes de licenciatura participantes en un programa de investigación de verano organizado por el Laboratorio de Políticas Transfronterizas de Arizona State University.

\section{Resultados}

El escenario contrafactual del que disponemos para el análisis de las respuestas de las ciudades fronterizas a la pandemia del COVID-19 es limitado. Sin embargo, como observa Collins-Dogrul (2012), aunque la cooperación transfronteriza en el tema de salud es un proceso difícil e imperfecto, la evidencia indica una participación substancial y sostenida de actores locales en la atención de problemas de salud fronterizos, particularmente en acciones de intercambio de datos epidemiológicos sobre enfermedades infecciosas, manejo de pacientes móviles y la transferencia de conocimiento y tecnología (Lobato \& Cegielski, 2001; Rosales et al., 2016; Schneider et al., 2004; Weinberg et al., 2003).

Como se indicó arriba, las acciones tomadas en el contexto de la pandemia del COVID-19 pueden ser agrupadas en dos categorías. Las acciones de contención o epidemiológicas tuvieron como propósito detener la propagación del virus mediante la reducción de las tasas de contagio. Las acciones de mitigación o no epidemiológicas, 
por otro lado, estuvieron dirigidas a atenuar el impacto del confinamiento sobre el empleo, la interacción social y el funcionamiento de los negocios y las instituciones y, en general, la disrupción de las actividades cotidianas de individuos, familias, empresas y organizaciones sociales.

Por lo que respecta a las acciones de contención, nuestros resultados indican que durante la emergencia creada por el COVID-19 la acción local se centró en la implementación de las declaratorias de emergencia federales y estatales. Como se muestra en la Figura 1, cerca de la mitad de las acciones emprendidas por las ciudades en ambos lados de la frontera se enfocaron en la prevención y en lograr el "aplanamiento de la curva” lo más rápidamente posible mediante la reducción de las tasas de transmisión del virus. Las medidas más utilizadas para el aplanamiento de la curva fueron el confinamiento domiciliario, el cierre de negocios, la prohibición de reuniones multitudinarias y el uso obligatorio de cubiertas faciales. Para asegurar la efectividad de estas medidas, su aplicación se acompañó de la vigilancia y la coerción en ambos lados de la frontera, pero especialmente en las ciudades mexicanas donde las acciones de cumplimiento de la ley fueron tres veces más frecuentes. De acuerdo con las fuentes consultadas, las infracciones más comunes fueron el incumplimiento del protocolo para la operación de negocios no esenciales, la inobservancia del confinamiento y de las restricciones a la congregación en grandes grupos, por lo que se impusieron multas a los infractores. Todas estas medidas fueron codificadas en las declaratorias de emergencia adoptada por la mayoría de las ciudades incluidas en la muestra.

Figura 1. Áreas de intervención local para contener la pandemia y mitigar el impacto de las acciones de contención

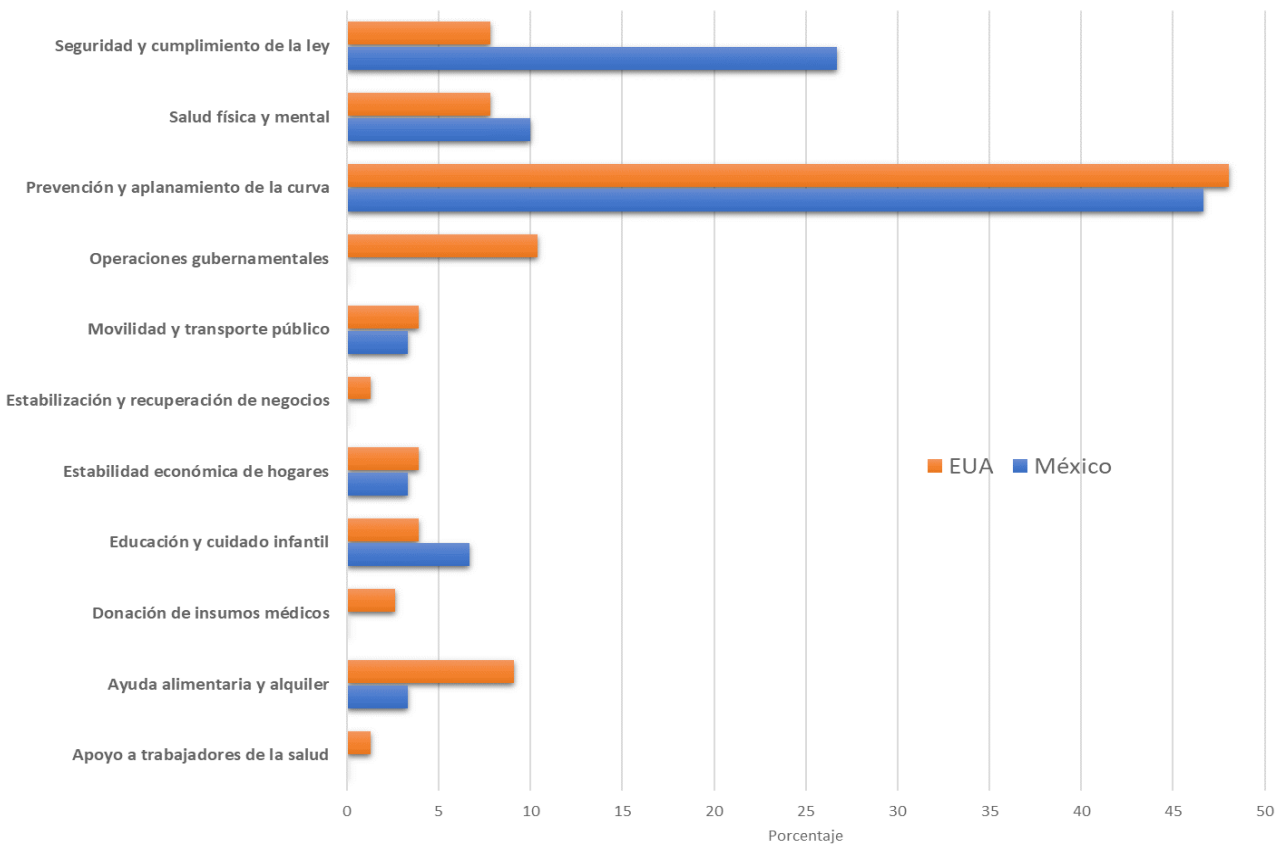

Fuente: TransborderLab, 2020 
En las ciudades mexicanas también fue común el establecimiento de estaciones sanitarias en los cruces fronterizos y dentro de la ciudad. En los cruces fronterizos, personal de salud realizaba tomas de temperatura, aplicaba desinfectantes en manos de peatones y requería a las personas que cruzaran por túneles desinfectantes antes de entrar a México. En esta área hay que resaltar las acciones de toma de temperatura a migrantes mexicanos deportados en diferentes puertos fronterizos. En las ciudades estadounidenses estas actividades no fueron reportadas, pero sí los eventos masivos de tamizaje para la detección del coronavirus. En el lado estadounidense también se registraron acciones de solidaridad con los trabajadores de la salud, así como donaciones de equipo de protección.

Por lo que toca a las acciones de mitigación de los efectos de las medidas para contener la propagación de la pandemia, destacan las realizadas para atender las necesidades de poblaciones social o económicamente vulnerables, como los programas de movilidad y entrega de comida y agua a personas de edad avanzada, la gratuidad del transporte público, la exención de pago de servicios o las prohibiciones para el desalojo de familias con dificultades para pagar el alquiler de su casa o departamento. Las acciones locales en el área de protección contra desalojos de la vivienda y asistencia alimentaria fueron más comunes en el lado estadounidense de la frontera, mientras que en el lado mexicano lo fueron las acciones de ayuda alimentaria o el pago adelantado de cuatro meses de pensión a adultos mayores y personas discapacitadas.

Otro renglón de actividad no epidemiológica fueron las acciones para la estabilización y eventual recuperación de empresas locales, sobre todo micro y pequeños negocios. Destacan las campañas para informar de los programas de apoyo en el pago de nóminas ofrecidos por el gobierno federal de los Estados Unidos, y en menor frecuencia las acciones de asistencia apoyadas en recursos locales.

\section{Pares de ciudades fronterizas}

La Tabla 2 presenta un panorama de las acciones por pares de ciudades fronterizas. Todas las acciones fueron jerarquizadas y estandarizadas usando una escala de 1-3 donde 1 representa la frecuencia más baja y 3 representa la frecuencia más alta según las fuentes analizadas en cada ciudad. La última columna de la Tabla 2 resume la relevancia de cada clase de acción para el conjunto de ciudades analizadas.

Las campañas informativas y las acciones de vigilancia para que la población fronteriza se quedara en casa fueron las medidas más comunes de contención implementadas en todos los pares de ciudades, aunque las acciones de vigilancia fueron más frecuentes en el lado mexicano. La prohibición de reuniones en grandes grupos, incluidos eventos deportivos y fiestas fue también frecuentemente reportada. De acuerdo con las fuentes consultadas, la vigilancia del cumplimiento de este tipo de acciones fue más directa y rigurosa en las ciudades mexicanas, donde se hizo uso frecuente de la policía y de multas para sancionar a quienes seguían congregándose en grupos grandes, particularmente en celebraciones y fiestas.

Todas las ciudades también emitieron algún tipo de declaratoria de emergencia al proclamar la obligatoriedad de acciones que limitaban la movilidad y la congregación, junto con otras medidas de contención como el cierre de escuelas, parques y oficinas gubernamentales. Además, se estableció el uso obligatorio de cubrebocas, el distanciamiento físico y la suspensión de actividades económicas no esenciales. 
En varias ciudades mexicanas se tomaron medidas para contener el riesgo asociado con los viajes no esenciales de personas desde Estados Unidos. En Mexicali y Ciudad Acuña, por ejemplo, se establecieron puestos de revisión en la frontera, mientras que en Nogales se instalaron estaciones de desinfección en las garitas para pasajeros y peatones. Muy pocas ciudades implementaron tamizajes masivos o mapeo de contactos. Este tipo de acciones solo se registró en las ciudades estadounidenses, destacan El Paso, Laredo, Nogales y Yuma.

Tabla 2. Acciones locales de contención de la epidemia y de mitigación de su impacto por pares de ciudades fronterizas

\begin{tabular}{|c|c|c|c|c|c|c|c|c|c|}
\hline Acciones locales & 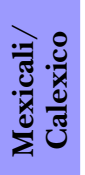 & 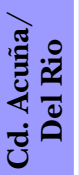 & 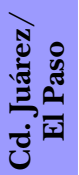 & 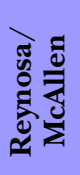 & 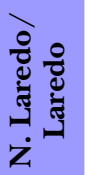 & 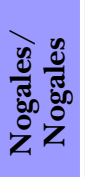 & 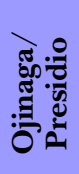 & 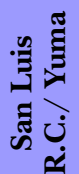 & 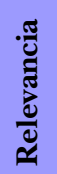 \\
\hline \multicolumn{10}{|c|}{ Acciones de contención } \\
\hline $\begin{array}{l}\text { Vigilancia confinamiento/ } \\
\text { Quédate en casa }\end{array}$ & 3 & 3 & 3 & 3 & 3 & 2 & 3 & 3 & 2.9 \\
\hline Declaración de emergencia & 2 & 2 & 2 & 2 & 3 & 1 & 2 & 3 & 2.1 \\
\hline Cierre de escuelas & 2 & 2 & 3 & 2 & 2 & 2 & 2 & 2 & 2.1 \\
\hline $\begin{array}{l}\text { Cumplimiento distanciamiento } \\
\text { social }\end{array}$ & 1 & 3 & 1 & 1 & 3 & 2 & 1 & 3 & 1.9 \\
\hline $\begin{array}{l}\text { Cierre de oficinas } \\
\text { gubernamentales }\end{array}$ & 2 & 2 & 2 & 2 & 2 & 2 & 2 & 2 & 2.0 \\
\hline $\begin{array}{l}\text { Mandato de uso de cubrebocas/ } \\
\text { vigilancia }\end{array}$ & 3 & 1 & 2 & 1 & 1 & 3 & 2 & 3 & 2.0 \\
\hline $\begin{array}{l}\text { Prohibición de reuniones en } \\
\text { grupos grandes }\end{array}$ & 3 & 3 & 3 & 2 & 3 & 3 & 1 & 3 & 2.6 \\
\hline $\begin{array}{l}\text { Mandato de cierre de negocios/ } \\
\text { vigilancia }\end{array}$ & 2 & 3 & 2 & 1 & 2 & 2 & 1 & 2 & 1.9 \\
\hline $\begin{array}{l}\text { Desaliento viajes transfronterizos } \\
\text { no esenciales }\end{array}$ & 2 & 3 & 1 & 1 & 1 & 3 & 2 & 1 & 1.8 \\
\hline $\begin{array}{l}\text { Prueba de contagio y mapeo de } \\
\text { contactos }\end{array}$ & 1 & 1 & 2 & 1 & 2 & 2 & 1 & 3 & 1.6 \\
\hline \multicolumn{10}{|c|}{ Acciones de mitigación } \\
\hline $\begin{array}{l}\text { Provisión de alimentos a grupos } \\
\text { vulnerables }\end{array}$ & 1 & 2 & 2 & 3 & 3 & 3 & 1 & 1 & 2.0 \\
\hline $\begin{array}{l}\text { Recuperación/reapertura } \\
\text { económica }\end{array}$ & 3 & 1 & 1 & 1 & 2 & 3 & 1 & 3 & 1.9 \\
\hline $\begin{array}{l}\text { Educación para la salud/cuidados } \\
\text { primarios }\end{array}$ & 1 & 3 & 3 & 1 & 1 & 1 & 1 & 3 & 1.8 \\
\hline Preparación/resiliencia & 2 & 1 & 1 & 2 & 2 & 1 & 1 & 1 & 1.4 \\
\hline Otras acciones no-epidemiológicas & 2 & 1 & 1 & 2 & 1 & 1 & 1 & 3 & 1.5 \\
\hline
\end{tabular}

Fuente: TransborderLab, 2020 
Las acciones de mitigación fueron menos prevalentes en general. Dentro de esta categoría destacan, por su mayor frecuencia, la distribución y entrega de alimentos y agua a población de la tercera edad y a familias e individuos afectados por la disminución de ingresos durante la pandemia. Aparte de los apoyos en alimentos, también se observaron, aunque con menos frecuencia, medidas para evitar los desalojos de viviendas o el corte de servicios públicos por falta de pago. Estas acciones fueron más comunes en las ciudades del río Bravo, aunque principalmente en el lado estadounidense.

El desplome de la actividad económica, principalmente en el comercio y los servicios, alentó esfuerzos locales para la recuperación y reapertura de la economía en ambos lados de la frontera. Las acciones más frecuentes fueron el ofrecimiento de talleres y campañas informativas de los programas de asistencia federal en Estados Unidos o el ofrecimiento de subvenciones a negocios pequeños como sucedió en Nogales, Arizona. Algunas ciudades, aunque en menor medida, también reportaron acciones encaminadas a elevar su resiliencia y grado de preparación ante futuras emergencias.

\section{Cooperación transfronteriza}

La epidemia hizo patente los riesgos y vulnerabilidades que confrontan las ciudades de la frontera a la vez que subrayó la necesidad de la cooperación transfronteriza. Tres situaciones detectadas mediante este análisis son particularmente ilustrativas.

La primera de estas situaciones se derivó del cierre parcial de la frontera anunciado el 21 de marzo de 2020 por el gobierno de Estados Unidos. Aunque el cierre fue anunciado como una medida bilateral para contener la propagación del virus al restringir la movilidad transfronteriza a solo viajes esenciales; en términos prácticos la medida excluyó del cruce fronterizo a los nacionales mexicanos residentes de la frontera, mientras que mantuvo sin cambio la prerrogativa de cruce de ciudadanos y residentes legales de Estados Unidos (Department of Homeland Security [DHs], 2020a). Aunque las estadísticas oficiales indican un desplome del número de cruces, la medida no canceló el riesgo de transmisión del coronavirus a través de la frontera. De hecho, luego de la abrupta contracción de los cruces en el mes de abril (cerca de 60\%, comparado con marzo), el nivel de movilidad se ha recuperado consistentemente a pesar de que el cierre se ha prorrogado en forma consecutiva en varias ocasiones sin cambios en los criterios de operación (Bureau of Transportation Services [втs], 2020). El movimiento constante de ciudadanos estadounidenses o trabajadores transmigrantes fue un factor de riesgo sanitario reportado en las fuentes analizadas. Por una parte, los días festivos y de asueto en ambos lados de la frontera continuaron generando flujos masivos de residentes de Estados Unidos hacia México, lo que elevó la preocupación por su impacto sobre la propagación del virus. Por otra parte, los jornaleros agrícolas y otros trabajadores transmigrantes empleados en actividades esenciales en Estados Unidos continuaron cruzando diariamente a laborar y retornar a México sin protocolos de control y monitoreo sanitario adecuados. Aunque el impacto de la movilidad de visitantes y de trabajadores transfronterizos sobre el número de infecciones y muertes en ambos lados de la frontera no ha sido evaluado, algunos reportes identificaron a la población que cosecha y procesa alimentos como un grupo de alto riesgo de infección por COVID-19, mientras que otros destacan a la población de origen mexicano en Estados Unidos como uno de los grupos más afectados por el 
COVID-19 en ese país (CDC, 2020; California Department of Public Health [CDPH], 2020; Thomas, 2020,). La movilidad facilitada por un cierre asimétrico de la frontera generó preocupación y malestar, lo cual se materializó en protestas y otras expresiones de frustración, sin que derivara inmediatamente en protocolos y acciones coordinadas de prevención a nivel local (CDC, 2020; Embajada y Consulados de Estados Unidos en México, 2020).

La segunda situación fue producto de una serie de medidas migratorias anunciadas por el gobierno estadounidense con implicaciones potenciales para la salud fronteriza. Una de estas medidas fue el programa de repatriación aérea que acordaba hacer pruebas de tamizaje de COVID-19 y el uso de cubrebocas de migrantes deportados durante el viaje de retorno a la Ciudad de México (DHs, 2020c). Otra es la deportación expedita de migrantes bajo las provisiones del título 42, sección 265 del código federal de Estados Unidos ordenada por el Departamento de Salud por razones de salud pública y ejecutada por CBP (U.s. Custom and Border Protection) en la frontera. La motivación del programa de repatriación aérea era desalentar el retorno de migrantes mexicanos a Estados Unidos y reducir el riesgo de exposición al COVID-19, "de los agentes de migración en puertos fronterizos, los agentes de la patrulla fronteriza y el pueblo americano" (DHs, 2020c). No obstante, diversas organizaciones de derechos humanos e instituciones académicas advirtieron que свР incumplió este acuerdo al realizar la repatriación de migrantes directamente en los puertos fronterizos. Esto, sumado a las deportaciones de migrantes indocumentados detenidos bajo las provisiones del Título 42, amplificaron el riesgo de propagación del virus en las ciudades de la frontera. De marzo a julio de 2020 las autoridades migratorias de Estados Unidos expulsaron 137536 personas por los cruces fronterizos con México, de los cuales 106000 fueron deportados bajo las provisiones del Título 42 (DHs, 2020b). De nuevo, la falta de protocolos para el control sanitario de la población migrante y el incumplimiento de acuerdos causó alarma y derivó en señalamientos contra las autoridades migratorias de Estados Unidos por contribuir a la diseminación del COVID-19 en la frontera (El Colegio de la Frontera Norte [El Colef], 2020b; Kassie \& Marcolini, 2020; Washington Office on Latin America [wola], 2020).

La tercera situación involucra a las organizaciones de asistencia humanitaria a la población migrante que operan en la frontera. Estas organizaciones incluyen comedores, albergues, oficina de asistencia legal y otras que repentinamente se enfrentaron con la necesidad urgente de desarrollar recursos y protocolos para atender a una población de alto riesgo. Aunque algunos albergues establecieron protocolos sanitarios en coordinación con las autoridades locales de salud, incluida la toma de temperatura y saneamiento a solicitantes de servicio, varias organizaciones reportaron niveles de demanda y condiciones de hacinamiento que impedían la aplicación de medidas de "sana distancia" dentro de sus instalaciones. Algunos centros de atención se vieron forzados a cerrar temporalmente, rechazar a solicitantes de asistencia o reducir sus servicios. En Nogales, Sonora, por ejemplo, el comedor para migrantes más importante de la ciudad comenzó a ofrecer alimentos "para llevar" con el objeto de minimizar el contacto de su personal con la población migrante. El análisis detectó que, a pesar de las medidas adoptadas, varios albergues en las ciudades fronterizas estudiadas reportaron brotes de COVID-19 entre la población residente. La elevación del riego causada por la población migrante llevó a que Ciudad Juárez estableciera en junio el primer "hotel filtro" en la frontera, parte central de un operativo que ofrecía servicios de salud y mantenía en confinamiento a los migrantes por 15 días antes de 
ser canalizados a alguno de los albergues de la ciudad (Organización Internacional para las Migraciones [oIm], 2020b; Paho, 2012). La habilitación de este mecanismo en Ciudad Juárez, y posteriormente en Tijuana, fue una medida de emergencia catalizada por la ausencia de protocolos efectivos de protección de la salud de la población migrante (оIм, 2020a, 2020b).

Aunque las comunidades fronterizas confrontaron niveles de riesgo y vulnerabilidad elevados derivados de su condición limítrofe, las acciones que involucraron alguna forma de cooperación transfronteriza fueron pocas y limitadas. En general, los casos observados de coordinación y colaboración entre ciudades corresponden a acciones restringidas y efímeras forzadas por el agravamiento de los indicadores de la pandemia, y solo después de que los sistemas locales de salud alcanzaron puntos críticos en su operatividad. En el caso de Mexicali y el Valle Imperial en la frontera de Baja California, por ejemplo, el catalizador fue el aumento del número de casos positivos y muertes en ambos lados de la frontera y la saturación de hospitales en el sur de California a finales de mayo de 2020. En Ciudad Acuña, Coahuila, y Rio Bravo, Texas, los alcaldes también acordaron colaborar para desalentar los viajes de personas con doble nacionalidad durante los días festivos de mayo y julio. Una negociación similar se observó en la región Sonora-Arizona, aunque esta se dio entre autoridades estatales.

\section{Discusión}

Los factores que están detrás del reducido número de acciones transfronterizas dirigidas a la contención del COVID-19 o la mitigación de impactos secundarios son diversos y complejos, y algunos de ellos se manifestaron en los datos recogidos por esta investigación.

A nivel funcional, un factor que limita el desarrollo de la cooperación son las limitaciones legales y las disparidades financieras y tecnológicas existentes entre ambos lados de la frontera. La actuación local en materia de salud y en otras áreas de importancia para las ciudades de la frontera tiene como contra fondo, políticas y un sistema de gestión regido por principios de soberanía y salvaguarda del interés nacional. Tanto Estados Unidos como México protegen su derecho soberano sobre el manejo de los asuntos concernientes a la frontera y dejan pocos espacios para las acciones descentralizadas que involucran la cooperación transfronteriza. Aun a pesar de que históricamente los temas de salud pública han ocupado uno de esos espacios, las políticas de salud para la frontera México-Estados Unidos son dictadas por los gobiernos federales respectivos, mientras que las agendas y programas sectoriales son manejados principalmente por los gobiernos estatales. Como resultado, las preferencias y voces de los actores locales fronterizos tienden a ocupar una posición marginal dentro del espacio de decisiones que han producido las acciones de contención de la pandemia y de mitigación de sus impactos secundarios.

También a nivel funcional, la incertidumbre que rodeó la pandemia forzó una mentalidad precautoria e introspectiva entre las autoridades políticas y de salud pública locales. Frente a la magnitud del riesgo y sus consecuencias, la estrategia más segura de contener la pandemia localmente es la coordinación con las autoridades de salud federales y estatales en cada país, lo que hace de la cooperación transfronteriza un concepto secundario dentro de las prioridades de cada condado o municipio fronterizo. De hecho, algunas de las acciones epidemiológicas transfronterizas más significativas 
involucraron a los departamentos estatales de salud mediante la activación de los protocolos de coordinación binacional existentes. Uno de esos protocolos es el mecanismo para la notificación de casos de enfermedades infecciosas, que facilita el intercambio de información y activa un sistema de transferencia de pacientes (El Colef, 2020a). La crisis que generó la llegada continua de ambulancias a los puertos fronterizos en el estado de California fue el catalizador de un sistema para ordenar la transferencia de pacientes por las garitas que conectan a Mexicali y al Valle Imperial.

A nivel operativo, las acciones coordinadas para la contención de la pandemia hubieran requerido un entendimiento similar y sincronizado de la seriedad del riesgo para la salud pública de ambos lados de la frontera, una condición difícil de lograr aun dentro de las fronteras de cada país. Además, para que las ciudades de la frontera hubieran podido diagnosticar y pronosticar la severidad de la pandemia en términos transfronterizos, la información sobre casos, contagios, letalidad y mortalidad por COVID-19 tendría que estar homologada metodológicamente y conceptualmente, y tener los datos disponibles en tiempo real en ambos lados de la frontera. Como se sabe, la severa fragmentación de los sistemas de salud en México y Estados Unidos hacen extremadamente difícil crear un registro confiable y unificado de las tasas de infección y mortalidad a escala nacional, lo que dificulta aún más la comparación directa entre países y entre escalas dentro de cada país.

No puede omitirse el rol de factores estructurales como las políticas federales hacia la frontera que al momento del brote del COVID-19 se encontraban en un momento único como resultado de la circunstancia política en cada país. En el caso de Estados Unidos, el gobierno de Donald Trump había impulsado una política soberanista y de distanciamiento que reforzaba narrativas de separación y representaba a México como un riesgo para la seguridad nacional (Da Silva, 2020). Aunque es posible que estas narrativas no tuvieran eco en las ciudades de la frontera estadounidense, su implicación más significativa desde el punto de vista de la gestión de asuntos fronterizos es que la no alineación con las posiciones de Washington eleva los costos políticos y económicos de las acciones descentralizadas. En el caso de México, las políticas de austeridad impuestas por la administración de Andrés Manuel López Obrador debilitaron las estructuras tradicionales para la cooperación transfronteriza como las actividades de apoyo realizadas por las oficinas consulares mexicanas cercanas a la frontera. Es probable que la acción local también haya sido afectada por la ideologización y politización de la pandemia, principalmente en Estados Unidos (Shear \& Dickerson, 2020). Como resultado de ello, las respuestas de los estados fronterizos estadounidenses variaron substancialmente, y en algunos momentos en sentido opuesto. Por ejemplo, una de las acciones iniciales del gobierno del estado de Arizona fue emitir una orden ejecutiva que prohibía a las ciudades actuar por su cuenta con la restricción de la operación de negocios o que se hiciera obligatorio el uso de cubrebocas (Arizona Medical Association, 2020; KTAR-NEWS, 2020). Estas acciones contrastan abiertamente con las medidas más estrictas adoptadas por los estados de California y Nuevo México. También llevaron al agravamiento de la pandemia en algunas ciudades de la frontera y, en última instancia, estimularon acciones locales que exigían el cambio en las políticas contra la pandemia (ABC15.com staff, 2020).

La imposibilidad de la acción descentralizada en este escenario es ilustrada por la respuesta de las ciudades fronterizas ante el cierre asimétrico de la frontera como principal herramienta para contener la propagación del coronavirus entre los dos países. Al mantener la movilidad transfronteriza de ciudadanos y residentes de 
Estados Unidos, el cierre de la frontera excluyó de la propagación a una parte de la población fronteriza, pero dejó a todos igualmente expuestos al riesgo de infección. Esto motivó algunas acciones de protesta por grupos de ciudadanos en México que exigían restricciones al cruce desde Estados Unidos para contener la propagación del virus (Shear \& Dickerson, 2020). En Nogales, Sonora, un grupo de personas obstruyó temporalmente el cruce y demandó que las autoridades aduanales de México impidieran viajes no esenciales desde Arizona. En Sonoyta, Sonora, un grupo de residentes impidió el tránsito de viajeros hacia Puerto Peñasco, un destino turístico regular para muchas familias de Arizona y algunas de las cuales poseen casas de playa en esa localidad. También estimuló acciones por parte de las autoridades locales como el establecimiento de estaciones de desinfección, puestos de información y verificación de temperatura por personal médico en los cruces fronterizos de varias de las ciudades mexicanas incluidas en el análisis (Prendergast, 2020). En Calexico y Laredo, por otro lado, se iniciaron campañas de información para desalentar el cruce transfronterizo, algunas veces en concertación con autoridades mexicanas. Las autoridades de Mexicali y de Imperial Valley, por ejemplo, acordaron coordinar campañas de información para disminuir los cruces fronterizos durante días festivos y establecieron controles de verificación de viajes esenciales en la frontera.

Todas estas acciones son medidas de contención que reflejan el carácter complejo y contradictorio de la vida en la frontera. En primer lugar, son una expresión de la imposibilidad local de incidir sobre las políticas que regulan la movilidad transfronteriza. En segundo lugar, hacen evidente los enfoques asimétricos de la salud pública entre ambos países, ya que, en México, la salud es un asunto del dominio público, mientras que en Estados Unidos es fundamentalmente un asunto privado. Por último, también refleja la politización de la pandemia en la sociedad estadounidense que se encuentra profundamente dividida sobre la legitimidad de las acciones para controlar la pandemia y que justifica que muchos ciudadanos rechacen medidas de protección como la sana distancia y el quédate en casa que tiene mayor aceptación en México (National Public Radio [NPR], 2020).

\section{Conclusiones}

La pandemia del COVID-19 ha alterado rápidamente las formas de vida urbana en todo el mundo y las ciudades de la frontera México-Estados Unidos no han quedado exentas de estas transformaciones. Al considerar su condición de espacios limítrofes y el impacto desproporcionado de la pandemia en algunas de ellas, es importante comprender cómo las dinámicas propias de las ciudades fronterizas influyen las respuestas locales a la pandemia y su efectividad, y cómo la pandemia trastorna las dinámicas sociales e institucionales características de los espacios de frontera. Reconocemos que estamos al comienzo de una comprensión plena y profunda de la interacción entre COVID-19 y comunidades fronterizas, pero hay necesidad de documentar - aunque sea de manera preliminar- eventos y procesos que hacen palpables las complicadas interacciones sociales que producen la adyacencia de asimetrías y complementariedades entre ciudades de la frontera México-Estados Unidos.

Sin duda, la propagación del COVID-19 a través de la frontera tiene entre sus vehículos de transmisión más importantes la fuerte interacción que producen mercados 
de trabajo y de consumo altamente integrados, así como las relaciones producidas por los arreglos familiares y modos de vida transfronterizos comunes en la región. Empero, también es claro que medidas unilaterales y centristas como el cierre de la frontera no aminoran el riesgo sanitario, y sí pueden contribuir a su reproducción y aun amplificarlo.

Sin embargo, y a pesar del historial de colaboración y grado de institucionalización de las redes de cooperación transfronteriza en el ámbito de la salud, es obvio que estas estructuras se quedan cortas frente a la magnitud y complejidad de la emergencia creada por la pandemia del COVID-19 en las ciudades de la frontera. A pesar de que instancias como los Consejos Binacionales de Salud podrían haber sido instrumentos lógicos en la articulación de respuestas transfronterizas a la pandemia, la información recopilada no registra acciones significativas que involucre a estos actores locales. Por lo que toca a las acciones epidemiológicas, nuestros resultados indican que la acción local ha sido principalmente de acompañamiento en la implementación de acciones centralizadas, ya sean estas medidas tomadas por las autoridades nacionales de salud o por las autoridades estatales. El sistema de salud pública en Estados Unidos y México da a los estados jurisdicción sobre la planeación y coordinación de políticas para el manejo de emergencias sanitarias. Tanto en México como en Estados Unidos, los gobiernos locales desempeñan un papel importante en la protección de la salud pública a través de una serie de actividades regulatorias. Por ejemplo, mediante programas de inspección y sistemas de licenciamiento, los condados y municipios se aseguran de que los negocios establecidos dentro de su jurisdicción operen conforme a normas de seguridad y salubridad adecuadas. También intervienen para que los habitantes no se involucren en conductas individual o colectivamente riesgosas mediante sistemas de vigilancia sanitaria o la imposición de confinamiento de personas que padecen enfermedades infecciosas. Como resultado, los gobiernos estatales de la frontera asumieron el papel principal en el diseño e implementación de la estrategia para la contención y la mitigación de la pandemia, mientras que los gobiernos locales concentraron su actividad en amplificar los programas estatales y en la vigilancia del cumplimiento de las medidas incluidas en las declaratorias de emergencia.

En suma, la emergencia creada por el COVID-19 está proyectando un escenario dual en materia de gobernanza transfronteriza. Por un lado, la pandemia ha revelado la debilidad de las estructuras locales para la cooperación transfronteriza en materia de salud ya que los gobiernos nacionales y estatales continúan dominando los espacios de decisión y los actores locales son agentes subsidiarios dentro de este espacio. Esto es particularmente preocupante cuando los actores nacionales divergen en prioridades y estrategias, y encasillan a las comunidades fronterizas en una suerte de trampa territorial y política que asume que el principio y el fin de relaciones sociales significativas para estas comunidades coinciden con la línea internacional (Agnew, 1994). Por otro lado, la situación creada por la pandemia podría ser vista como una oportunidad para promover nuevas prácticas institucionales y la renegociación de los espacios para la cooperación transfronteriza en materia de salud. Es posible que una vez que la magnitud e implicaciones del cierre de la frontera y otras medidas centristas se expresen plenamente sea necesario crear condiciones para que los actores locales asuman una posición más proactiva y estratégica que enfatice la resiliencia y la preparación frente a choques externos futuros que pudieran afectar dinámicas y relaciones transfronterizas esenciales para la vida local. 
Un ejemplo de lo que es posible para escapar de la trampa territorial es el modelo de burbujas urbanas propuesto por Detsky y Bogoch (2020) para algunas provincias canadienses, y que tiene como propósito mantener la movilidad entre ciudades aledañas. Aplicado a la frontera, el modelo podría ser la base de la creación de burbujas binacionales que hubieran servido para mantener la movilidad trasfronteriza, y con ello las relaciones económicas y sociales esenciales, cuando dos ciudades vecinas mostraran una reducción consistente en el número de casos de COVID-19. Los mecanismos de gobernanza necesarios para la implementación de soluciones como estas, sin embargo, necesitan ser construidos.

\section{Agradecimiento}

Los autores agradecen a Lisa Jantzie, Linh Luong, Nicole Maestas, Tarek Tharwat, Amanda Weissgerber y Lu Zhou, estudiantes de Arizona State University y becarios del Laboratorio de Políticas Transfronterizas, por su asistencia en esta investigación.

\section{Referencias}

ABC15.com staff. (2020). Nogales mayor asks Gov. Ducey for authority to require city's residents to wear masks. ABC 15 Arizona. https://www.abc15.com/news/ region-central-southern-az/nogales/city-of-nogales-mayor-asking-gov-ducey-forauthority-to-require-masks

Agnew, J. (1994). The territorial trap: the geographical assumptions of international relations theory. Review of International Political Economy, 1(1), 53-80. http://www. jstor.org/stable/4177090

Álvarez, R. (2012). Reconceptualizing the space of the Mexico-u.s. borderline. En T. Wilson \& H. Donnan (Eds.), A companion to border studies (pp. 538-556). Blackwell Publishing.

Anderson, J., Gerber, J. \& Foster, L. (2008). Fifty years of change on the U.S.-Mexico border: growth, development, and quality of life. University of Texas Press.

Arizona Medical Association. (2020). Stay calm. Stay alert. Responding to the Coronavirus (COVID-19). Resources for physicians E patients. https://www.azmed.org/ page/Coronavirus

Arreola, D. D. (2010). The Mexico-us borderlands through two decades. Journal of Cultural Geography, 27(3), 331-351. https://doi.org/10.1080/08873631.2010.517621

Arreola, D. D. \& Curtis, J. R. (1993). The Mexican border cities: landscape anatomy and place personality. University of Arizona Press.

azdhs-admin. (2011). Building a healthier border. Arizona Department of Health Services. https://directorsblog.health.azdhs.gov/building-a-healthier-border/

Bureau of Transportation Services (втS). (2020). Border Crossing/Entry Data. https://www.bts.gov/browse-statistical-products-and-data/border-crossing-data/border-crossingentry-data

California Department of Public Health (CDPH). (2020). COVID-19 Race and ethnicity data https://www.cdph.ca.gov/Programs/CID/DCDC/Pages/COVID-19/ Race-Ethnicity.aspx 
Center for Disease Control and Prevention (CDC). (2020). Agriculture Workers and Employers Interim Guidance from $\mathrm{CDC}$ and the U.s. Department of Labor. https://www.cdc.gov/coronavirus/2019-ncov/community/guidance-agricultural-workers.html

Collins-Dogrul, J. (2006). Managing us-Mexico "border health": an organizational field approach. Social Science E Medicine, 63(12), 3199-3211. https://doi.org/10.1016/j. socscimed.2006.07.031

Collins-Dogrul, J. (2012). Tertius Iungens brokerage and transnational intersectoral cooperation. Organization Studies, 33(8), 989-1014. https://doi. org/10.1177\%2F0170840612445118

Da Silva, C. (2020, 20 de agosto). Trump claims border wall is helping stop spread of COVID-19 from Mexican city in 'South America'. Newsweek. https://www.newsweek.com/trump-border-wall-coronavirus-south-america-1526434

Detsky, A. S. \& Bogoch, I. I. (2020). COVID-19 in Canada: experience and response. JAMA, 324(8), 743-744. doi:10.1001/jama.2020.14033

Department of Homeland Security (DHS). (2020a). Fact Sheet: DHS measures on the border to limit the further spread of Coronavirus. https://www.dhs.gov/news/2020/08/14/factsheet-dhs-measures-border-limit-further-spread-coronavirus

Department of Homeland Security (DHS). (2020b). Nationwide enforcement encounters: Title 8 Enforcement actions and Title 42 expulsions. https://www.cbp.gov/news$\mathrm{room} / \mathrm{stats} / \mathrm{cbp}$-enforcement-statistics/title-8-and-title-42-statistics

Department of Homeland Security (DHS). (2020c). USBP E ICE Ramping up repatriation flights; effort aimed at reducing COVID-19 exposure in U.S. https://www.cbp. gov/newsroom/local-media-release/usbp-ice-ramping-repatriation-flights-effort-aimed-reducing-covid-19

El Colegio de la Frontera Norte (El Colef). (2020a, 16 de junio). Acciones implementadas ante COVID-19 en los estados de la región fronteriza México-Estados Unidos [Video]. YouTube. https://www.youtube.com/watch?v=rZ8rV_zaFdg\&ab_channel=ElColegiodelaFronteraNorte-ElColef

El Colegio de la Frontera Norte (El Colef). (2020b). Migrantes en albergues en las ciudades fronterizas del norte de México (Documentos de contingencia 2. Poblaciones vulnerables ante el COVID-19). https://www.colef.mx/wp-content/uploads/2020/04/ Albergues_w6.pdf

Embajada y Consulados de Estados Unidos en México. (2020). Como socios, vecinos y amigos, cooperamos en la respuesta ante el COVID19. https:/ / mx.usembassy.gov/es/ como-socios-vecinos-y-amigos-cooperamos-en-la-respuesta-ante-el-covid19/

Ganster, P. \& Collins, K. (2017). Binational cooperation and twinning: a view from the us-Mexican border, San Diego, California, and Tijuana, Baja California. Journal of Borderlands Studies, 32(4), 497-511. https://doi.org/10.1080/08865655.2016. 1198582

García-Pérez, H. (2007). Proyecto de seguimiento a la Asociación Fronteriza Mexicano-Estadounidense de Salud/United States-Mexico Border Health Association (AFMES/USMBHA). http://alamo.colson.edu.mx:8085/sitios/CESS/091020_frutosTrabajo/frutos_ archivos/2007_Garcia_ProyectoSeguimiento_AvanceInvest.pdf

García-Pérez, H. (2010). Health issues. North American Center for Transborder Studies-Arizona State University. https://doi.org/10.13140/RG.2.2.26679.85922 
Garza-Almanza, V. (2018). Fundación Binacional México-Estados Unidos para la Salud y el Ambiente: Crónica de un proyecto altruista: 1982-1991. Culcyt, 15(65), 8-33. http://erevistas.uacj.mx/ojs/index.php/culcyt/article/view/2661/2461

Gerber, J., Lara-Valencia, F. \& de la Parra, C. (2010). Re-Imagining the U.S.-Mexico border: policies toward a more competitive and sustainable transborder region. Global Economy Journal, 10(4). https://doi.org/10.2202/1524-5861.1681

Grimson, A. (2006). Cultures are more hybrid than identifications: A dialogue on borders from the Southern Cone. Latino Studies, 4(1), 96-119. https://doi. org/10.1057/palgrave.lst.8600177

Hataley, T. \& Leuprecht, C. (2018). Determinants of cross-border cooperation. Journal of Borderlands Studies, 33(3), 317-328. https://doi.org/10.1080/08865655.2018. 1482776

Homedes, N. \& Ugalde, A. (2003). Globalization and health at the United States-Mexico border. American Journal of Public Health, 93(12), 2016-2022. https://doi. org/10.2105/AJPH.93.12.2016

Instituto Nacional de Estadística y Geografía (Inegi). (2020). Censo de Población y Vivienda 2020. https://www.inegi.org.mx/datos/\#Areas_geograficas

Johnson, M. A. \& Niemeyer, E. D. (2008). Ambivalent landscapes: environmental justice in the US-Mexico borderlands. Human Ecology, 36(3), 371-382. https://doi. org/10.1007/s10745-008-9171-8

Kassie, E. \& Marcolini, B. (2020). How ICE exported the coronavirus. an investigation reveals how immigration and customs enforcement became a domestic and glo- bal spreader of COVID-19. https://www.themarshallproject.org/2020/07/10/how-ice-exported-the-coronavirus

Klatt, M. (2020). What has happened to our cross-border regions? Corona, unfamiliarity, and transnational border activism in the Danish-German border region. En C. Wille \& R. Kanesu (Eds.), Bordering in pandemic times: insighths into the COVID-19 lockdown (Borders in perspective 4, pp. 43-47): UniGR-Center for Border Studies. https://ubt.opus.hbz-nrw.de/opus45-ubtr/frontdoor/deliver/ index/docId/1428/file/UniGR-CBS_Borders+in+Perspective_thematic+issue_ Vol.+4.pdf

KTAR-NEWS. (2020). Ducey to give local leaders authority to institute mandatory mask policy. https://ktar.com/story/3297947/ducey-to-give-local-leaders-authority-to-institute-mandatory-mask-policy/

Lara-Valencia, F., Agulló, J., García-Pérez, H., Trillo, J. M. \& Oliveras, X. (2020). COVID-19 and cross-border mobility. An international survey of border experts. Arizona State University. https://doi.org/10.13140/RG.2.2.29353.72807

Lobato, M. N. \& Cegielski, J. P. (2001). Preventing and controlling tuberculosis along the U.S.-Mexico border. https://www.cdc.gov/MMWR/Preview/mmwrhtml/ rr5001a1.htm

National Public Radio (NPR). (2020). Lessons in handling the coronavirus Arizona could learn from Massachusetts. https://www.npr.org/2020/07/06/887925743/lessons-in-handling-the-coronavirus-arizona-could-learn-from-massachusetts

Organización Internacional para las Migraciones (огм). (2020a). Migrantes en alojamientos temporales en el norte de México reciben supervisión médica constante. https:/ / rosanjose.iom.int/site/es/noticia/migrantes-en-alojamientos-temporales-en-el-norte-de-mexico-reciben-supervision-medica 
Organización Internacional para las Migraciones (OIM). (2020b). OIM y socios ofrecen alojamiento digno en 'hotel filtro' para migrantes que necesitan pasar la cuarentena en el norte de México. https://rosanjose.iom.int/site/es/noticia/oim-y-sociosofrecen-alojamiento-digno-en-hotel-filtro-para-migrantes-que-necesitan-pasar-la

Panamerican Health Organization (Paho). (2012). United States-Mexico border area. https://www.paho.org/salud-en-las-americas-2012/index.php?option=com_content\&view=article\&id=63:united-statesmexico-border-area\&Itemid=173\&lang=en

Prendergast, C. (2020, 8 de mayo). Desinfectan con aerosol a quienes cruzan en carro de Arizona a Nogales, Sonora. La Estrella de Tucson. https://tucson.com/laestrella/frontera/desinfectan-con-aerosol-a-quienes-cruzan-en-carro-de-arizonaa-nogales-sonora/article_e36a50c0-914b-11ea-95f0-bb2df2906697.html

Rosales, C., Carvajal, S. \& De Zapien, J. (2016). Emergent public health issues in the US-Mexico border region. Frontiers Media. https://www.frontiersin.org/research-topics/3003/ emergent-public-health-issues-in-the-us-mexico-border-region

Schneider, E., Laserson, K. F., Wells, C. D. \& Moore, M. (2004). Tuberculosis along the United States-Mexico border, 1993-2001. Revista Panamericana de Salud Pública, 16(1), 23-34. https://scielosp.org/pdf/rpsp/2004.v16n1/23-34/en

Shear, M. D. \& Dickerson, C. (2020, 10 de agosto). Trump considers banning re-entry by citizens who may have coronavirus. The New York Times. https://www.nytimes. com/2020/08/10/us/politics/trump-coronavirus-border.html

Sohn, C. (2014). The border as a resource in the global urban space: a contribution to the cross-border metropolis hypothesis. International Journal of Urban and Regional Research, 38(5), 1697-1711. https://doi.org/10.1111/1468-2427.12071

TransborderLab. (2020). Effects of the COVID-19 Pandemic in Border Regions: Rapid Assessment and Bordering Prospects, Transborder Policy Lab, transborder.org

Thomas, N. (2020). Farm workers at extra risk for Covid-19, CDC says in new guidance. cNN. https://www.cnn.com/world/live-news/coronavirus-pandemic-06-02-20-intl/h_dd2956cc9999c985177da24ac662a09f

United States Census Bureau. (2020). 2015-2019 American community survey 5-year estimates. https://data.census.gov/cedsci/

Varady, R. \& Mack, M. (1995). Transboundary water resources and public health in the U.S.-Mexico border region. Journal of Environmental Health, 57 (8), 8-14.

Weinberg, M., Waterman, S., Álvarez Lucas, C., Carrion Falcon, V., Kuri Morales, P., Anaya, L., Peter, C., Escobar Gutiérrez, A., Ramirez-Gonzalez, J. E., Flisser, A., Bryan, R., Navarro Valle, E., Rodríguez, A., Alvarez, G., Rosales, C., Arias Ortiz, J., Landen, M., Vilchis, H., Lopez Leal, F. et al. (2003). The US-Mexico border infectious disease surveillance project: Establishing binational border surveillance. Emerging infectious diseases, 9(1), 97. https://dx.doi.org/10.3201/ eid0901.020047

World Health Organization (wHO). (2020). Timeline: WHO's response to COVID-19. https://www.who.int/emergencies/diseases/novel-coronavirus-2019/interactive-timeline

Wille, C. (2020). Border(ing)s in times of COVID-19. En C. Wille \& R. Kanesu (Eds.), Bordering in pandemic times: insighths into the COVID-19 lockdown (Borders in 
perspective 4, pp. 9-16). UniGR-Center for Border Studies. https://ubt.opus. hbz-nrw.de/opus45-ubtr/frontdoor/deliver/index/docId/1428/file/UniGR-CBS_Borders+in+Perspective_thematic+issue_Vol.+4.pdf

Washington Office on Latin America (wola). (2020). U.S. and Mexico must urgen- tly address impact of ongoing deportations and expulsions during COVID-19. https://www. wola.org/2020/05/usa-mexico-ongoing-deportations-expulsions-covid-19/

Francisco Lara-Valencia

Mexicano. Doctor en planeación urbana por la Universidad de Michigan. Profesor asociado en la School of Transborder Studies, de la Arizona State University, Estados Unidos. Líneas de investigación: desarrollo regional, sustentabilidad urbana, calidad de vida. Publicación reciente: Lara-Valencia, F. (2020). Transborder spaces and regional identity in North America: a comparative analysis. En G. Correa-Cabrera \& V. Conrad (Eds.), North American Borders in Comparative Perspective. University of Arizona Press.

Hilda García-Pérez

Mexicana. Doctora en epidemiología por la Universidad de Michigan. Investigadora del Departamento de Estudios de Población en El Colegio de la Frontera Norte, sede Nogales, México. Líneas de investigación: disparidades de salud en población femenina y adolescente en la región Sonora-Arizona. Publicación reciente: GarcíaPérez, H. \& Lara-Valencia, F. (2021). Association between neighborhood parks and leisure-time physical activity among adult Mexican women. Retos, 41, 544-554. 